\title{
DSpace@MIT
}

\author{
MIT Open Access Articles
}

\section{Search for the Photoexcitation of Exotic Mesons in the pil+lpil+)pil-) System}

The MIT Faculty has made this article openly available. Please share how this access benefits you. Your story matters.

Citation: Nozar, M. et al. "Search for the Photoexcitation of Exotic Mesons in the $\Pi+\Pi+\Pi-$

System.” Physical Review Letters 102.10 (2009) : 102002 (C) 2009 The American Physical Society.

As Published: http://dx.doi.org/10.1103/PhysRevLett.102.102002

Publisher: American Physical Society

Persistent URL: http://hdl.handle.net/1721.1/65363

Version: Final published version: final published article, as it appeared in a journal, conference proceedings, or other formally published context

Terms of Use: Article is made available in accordance with the publisher's policy and may be subject to US copyright law. Please refer to the publisher's site for terms of use. 


\section{Search for the Photoexcitation of Exotic Mesons in the $\pi^{+} \pi^{+} \pi^{-}$System}

M. Nozar, ${ }^{1}$ C. Salgado, ${ }^{1}$ D. P. Weygand, ${ }^{1}$ L. Guo, ${ }^{1, *}$ G. Adams $,{ }^{1} \mathrm{Ji} \mathrm{Li},{ }^{1}$ P. Eugenio, ${ }^{4}$ M. J. Amaryan,${ }^{31}$ M. Anghinolfi, ${ }^{21}$ G. Asryan, ${ }^{41}$ H. Avakian, ${ }^{1}$ H. Bagdasaryan, ${ }^{41,31}$ N. Baillie, ${ }^{40}$ J. P. Ball, ${ }^{6}$ N. A. Baltzell, ${ }^{35}$ S. Barrow ${ }^{4}$ M. Battaglieri, ${ }^{21}$ I. Bedlinskiy, ${ }^{23}$ M. Bektasoglu,${ }^{31, \dagger}$ M. Bellis, ${ }^{9}$ N. Benmouna, ${ }^{17}$ B. L. Berman, ${ }^{17}$ A. S. Biselli, ${ }^{3,15}$ L. Blaszczyk,${ }^{4}$ B. E. Bonner, ${ }^{33}$ S. Bouchigny, ${ }^{22}$ S. Boiarinov, ${ }^{23,1}$ R. Bradford, ${ }^{9}$ D. Branford,${ }^{14}$ W. J. Briscoe, ${ }^{17}$ W. K. Brooks, ${ }^{36}$ S. Bültmann, ${ }^{31}$ V. D. Burkert, ${ }^{1}$ C. Butuceanu, ${ }^{40}$ J. R. Calarco, ${ }^{29}$ S. L. Careccia ${ }^{31}$ D. S. Carman, ${ }^{1}$ B. Carnahan,${ }^{10}$ L. Casey, ${ }^{10}$ A. Cazes, ${ }^{35}$ S. Chen, ${ }^{4}$ L. Cheng, ${ }^{10}$ P. L. Cole, ${ }^{1,19}$ P. Collins, ${ }^{6}$ P. Coltharp,${ }^{4}$ D. Cords, ${ }^{1, \| l \mid}$ P. Corvisiero, ${ }^{21}$ D. Crabb,${ }^{39}$ H. Crannell, ${ }^{10}$ V. Crede, ${ }^{4}$ J. P. Cummings,${ }^{3}$ D. Dale, ${ }^{19}$ N. Dashyan, ${ }^{41}$ R. De Masi, ${ }^{11}$ R. De Vita, ${ }^{21}$ E. De Sanctis, ${ }^{20}$ P. V. Degtyarenko, ${ }^{1}$ H. Denizli,${ }^{32}$ L. Dennis,${ }^{4}$ A. Deur, ${ }^{1}$ K. V. Dharmawardane,${ }^{31}$ K. S. Dhuga,${ }^{17}$ R. Dickson, ${ }^{9}$ C. Djalali, ${ }^{35}$ G. E. Dodge, ${ }^{31}$ D. Doughty, ${ }^{12,1}$ M. Dugger, ${ }^{6}$ S. Dytman, ${ }^{32}$ O. P. Dzyubak, ${ }^{35}$ H. Egiyan, ${ }^{40,1, *}$ K. S. Egiyan,,${ }^{41, \| l l}$ L. El Fassi, ${ }^{5}$ L. Elouadrhiri, ${ }^{1}$ R. Fatemi, ${ }^{39}$ G. Fedotov, ${ }^{28}$ R. J. Feuerbach, ${ }^{9}$ T. A. Forest, ${ }^{19}$ A. Fradi, ${ }^{22}$ H. Funsten,,${ }^{40,|l l|}$ M. Garçon, ${ }^{11}$ G. Gavalian, ${ }^{29,31}$ N. Gevorgyan, ${ }^{41}$ G. P. Gilfoyle, ${ }^{34}$ K. L. Giovanetti, ${ }^{24}$ F. X. Girod,${ }^{11,1}$ J. T. Goetz, ${ }^{7}$ R. W. Gothe, ${ }^{35}$ K. A. Griffioen, ${ }^{40}$ M. Guidal, ${ }^{22}$ M. Guillo, ${ }^{35}$ N. Guler, ${ }^{31}$ V. Gyurjyan, ${ }^{1}$ C. Hadjidakis, ${ }^{22}$ K. Hafidi, ${ }^{5}$ H. Hakobyan,${ }^{41}$ C. Hanretty, ${ }^{4}$ J. Hardie,${ }^{12,1}$ N. Hassall, ${ }^{18}$ D. Heddle, ${ }^{1, \S}$ F. W. Hersman, ${ }^{29}$ K. Hicks, ${ }^{30}$ I. Hleiqawi, ${ }^{30}$ M. Holtrop, ${ }^{29}$ C. E. Hyde-Wright, ${ }^{31}$ Y. Ilieva, ${ }^{17}$ D. G. Ireland, ${ }^{18}$ B. S. Ishkhanov, ${ }^{28}$ E. L. Isupov, ${ }^{28}$ M. M. Ito, ${ }^{1}$ D. Jenkins, ${ }^{38}$ H. S. Jo, ${ }^{22}$ J. R. Johnstone, ${ }^{18}$ K. Joo, ${ }^{1,13}$ H. G. Juengst, ${ }^{31}$ N. Kalantarians, ${ }^{31}$ J. D. Kellie, ${ }^{18}$ M. Khandaker, ${ }^{2}$ W. Kim, ${ }^{25}$ A. Klein, ${ }^{31}$

F. J. Klein, ${ }^{10}$ M. Kossov, ${ }^{23}$ Z. Krahn, ${ }^{9}$ L. H. Kramer, ${ }^{16,1}$ V. Kubarovsky, ${ }^{1}$ J. Kuhn, ${ }^{3,9}$ S. E. Kuhn, ${ }^{31}$ S. V. Kuleshov, ${ }^{36}$ V. Kuznetsov, ${ }^{25}$ J. Lachniet, ${ }^{9,31}$ J. M. Laget, ${ }^{11,1}$ J. Langheinrich, ${ }^{35}$ D. Lawrence, ${ }^{27}$ K. Livingston, ${ }^{18}$ H. Y. Lu, ${ }^{35}$ M. MacCormick, ${ }^{22}$ N. Markov, ${ }^{13}$ P. Mattione, ${ }^{33}$ S. McAleer, ${ }^{4}$ B. McKinnon, ${ }^{18}$ J. W. C. McNabb, ${ }^{9}$ B. A. Mecking, ${ }^{1}$ S. Mehrabyan, ${ }^{32}$ M. D. Mestayer, ${ }^{1}$ C. A. Meyer,${ }^{9}$ T. Mibe,${ }^{30}$ K. Mikhailov, ${ }^{23}$ M. Mirazita, ${ }^{20}$ R. Miskimen, ${ }^{27}$ V. Mokeev, ${ }^{28,1}$ B. Moreno, ${ }^{22}$ K. Moriya, ${ }^{9}$ S. A. Morrow, ${ }^{22,11}$ M. Moteabbed, ${ }^{16}$ J. Mueller, ${ }^{32}$ E. Munevar,${ }^{17}$ G. S. Mutchler,${ }^{33}$ P. Nadel-Turonski, ${ }^{17}$ R. Nasseripour, ${ }^{16,35}$ S. Niccolai, ${ }^{17,22}$ G. Niculescu, ${ }^{30,24}$ I. Niculescu, ${ }^{17,24}$ B. B. Niczyporuk, ${ }^{1}$ M. R. Niroula, ${ }^{31}$ R. A. Niyazov, ${ }^{31,3}$ G. V. O'Rielly,${ }^{17}$ M. Osipenko, ${ }^{21,28}$ A. I. Ostrovidov ${ }^{4}$ K. Park, ${ }^{25, \|}$ E. Pasyuk, ${ }^{6}$ C. Paterson, ${ }^{18}$ S. Anefalos Pereira,${ }^{20}$ S. A. Philips, ${ }^{17}$ J. Pierce, ${ }^{39}$ N. Pivnyuk, ${ }^{23}$ D. Pocanic, ${ }^{39}$ O. Pogorelko, ${ }^{23}$ E. Polli, ${ }^{20}$ I. Popa,${ }^{17}$ S. Pozdniakov, ${ }^{23}$ B. M. Preedom,${ }^{35}$ J. W. Price, ${ }^{8}$ Y. Prok, ${ }^{39, \pi}$ D. Protopopescu, ${ }^{29,18}$ L. M. Qin, ${ }^{31}$ B. A. Raue,${ }^{16,1}$ G. Riccardi, ${ }^{4}$ G. Ricco, ${ }^{21}$ M. Ripani, ${ }^{21}$ B. G. Ritchie, ${ }^{6}$ F. Ronchetti, ${ }^{20}$ G. Rosner, ${ }^{18}$ P. Rossi, ${ }^{20}$ P. D. Rubin, ${ }^{34}$ F. Sabatié, ${ }^{11}$ J. Salamanca, ${ }^{19}$ J. P. Santoro, ${ }^{38,10,1}$ V. Sapunenko, ${ }^{1}$ R. A. Schumacher, ${ }^{9}$ V. S. Serov, ${ }^{23}$ Y. G. Sharabian, ${ }^{1}$ D. Sharov, ${ }^{28}$

N. V. Shvedunov, ${ }^{28}$ A. V. Skabelin, ${ }^{26}$ E. S. Smith, ${ }^{1}$ L. C. Smith, ${ }^{39}$ D. I. Sober, ${ }^{10}$ D. Sokhan, ${ }^{14}$ A. Stavinsky, ${ }^{23}$

S. S. Stepanyan, ${ }^{25}$ S. Stepanyan, ${ }^{12,1}$ B. E. Stokes, ${ }^{4}$ P. Stoler, ${ }^{3}$ I. I. Strakovsky, ${ }^{17}$ S. Strauch, ${ }^{35}$ M. Taiuti, ${ }^{21}$ D. J. Tedeschi ${ }^{35}$ U. Thoma, ${ }^{1, * *}$ A. Tkabladze, ${ }^{17, \dagger}$ S. Tkachenko, ${ }^{31}$ L. Todor, ${ }^{9, \dagger \dagger}$ M. Ungaro, ${ }^{3,13}$ M. F. Vineyard,${ }^{37,34}$ A. V. Vlassov, ${ }^{23}$ D. P. Watts, ${ }^{18,+\neq}$ L. B. Weinstein, ${ }^{31}$ M. Williams, ${ }^{9}$ E. Wolin, ${ }^{1}$ M. H. Wood, ${ }^{35,8}$ A. Yegneswaran, ${ }^{1}$ L. Zana, ${ }^{29}$ J. Zhang, ${ }^{31}$ B. Zhao, ${ }^{13}$ and Z. W. Zhao ${ }^{35}$

\section{(CLAS Collaboration)}

${ }^{1}$ Thomas Jefferson National Accelerator Facility, Newport News, Virginia 23606, USA
${ }^{2}$ Norfolk State University, Norfolk, Virginia 23504, USA
${ }^{3}$ Rensselaer Polytechnic Institute, Troy, New York 12180-3590, USA
${ }^{4}$ Florida State University, Tallahassee, Florida 32306, USA
${ }^{5}$ Argonne National Laboratory, Argonne, Illinois 60439, USA
${ }^{6}$ Arizona State University, Tempe, Arizona 85287-1504, USA
${ }^{7}$ University of California at Los Angeles, Los Angeles, California 90095-1547, USA
${ }^{8}$ California State University, Dominguez. Hills, Carson, California 90747, USA
${ }^{9}$ Carnegie Mellon University, Pittsburgh, Pennsylvania 15213, USA
${ }^{10}$ Catholic University of America, Washington, D.C. 20064, USA
${ }^{11}$ CEA-Saclay, Service de Physique Nucléaire, 91191 Gif-sur-Yvette, France
${ }^{12}$ Christopher Newport University, Newport News, Virginia 23606, USA
${ }^{13}$ University of Connecticut, Storrs, Connecticut 06269, USA
${ }^{14}$ Edinburgh University, Edinburgh EH9 3JZ, United Kingdom
${ }_{15}^{15}$ Fairfield University, Fairfield, Connecticut 06824, USA
${ }^{16}$ Florida International University, Miami, Florida 33199, USA
${ }^{17}$ The George Washington University, Washington, D.C. 20052, USA




\author{
${ }^{18}$ University of Glasgow, Glasgow G12 8QQ, United Kingdom \\ ${ }^{19}$ Idaho State University, Pocatello, Idaho 83209, USA \\ ${ }^{20}$ INFN, Laboratori Nazionali di Frascati, 00044 Frascati, Italy \\ ${ }^{21}$ INFN, Sezione di Genova, 16146 Genova, Italy \\ ${ }^{22}$ Institut de Physique Nucleaire ORSAY, Orsay, France \\ ${ }^{23}$ Institute of Theoretical and Experimental Physics, Moscow, 117259, Russia \\ ${ }^{24}$ James Madison University, Harrisonburg, Virginia 22807, USA \\ ${ }^{25}$ Kyungpook National University, Daegu 702-701, South Korea \\ ${ }^{26}$ Massachusetts Institute of Technology, Cambridge, Massachusetts 02139-4307, USA \\ ${ }^{27}$ University of Massachusetts, Amherst, Massachusetts 01003, USA \\ ${ }^{28}$ Moscow State University, General Nuclear Physics Institute, 119899 Moscow, Russia \\ ${ }^{29}$ University of New Hampshire, Durham, New Hampshire 03824-3568, USA \\ ${ }^{30}$ Ohio University, Athens, Ohio 45701, USA \\ ${ }^{31}$ Old Dominion University, Norfolk, Virginia 23529, USA \\ ${ }^{32}$ University of Pittsburgh, Pittsburgh, Pennsylvania 15260, USA \\ ${ }^{33}$ Rice University, Houston, Texas 77005-1892, USA \\ ${ }^{34}$ University of Richmond, Richmond, Virginia 23173, USA \\ ${ }^{35}$ University of South Carolina, Columbia, South Carolina 29208, USA \\ ${ }^{36}$ Universidad Técnica Federico Santa María, Casilla 110-V, Valparaíso, Chile \\ ${ }^{37}$ Union College, Schenectady, New York 12308, USA \\ ${ }^{38}$ Virginia Polytechnic Institute and State University, Blacksburg, Virginia 24061-0435, USA \\ ${ }^{39}$ University of Virginia, Charlottesville, Virginia 22901, USA \\ ${ }^{40}$ College of William and Mary, Williamsburg, Virginia 23187-8795, USA \\ ${ }^{41}$ Yerevan Physics Institute, 375036 Yerevan, Armenia \\ (Received 6 June 2008; published 12 March 2009)
}

A search for exotic mesons in the $\pi^{+} \pi^{+} \pi^{-}$system photoproduced by the charge exchange reaction $\gamma p \rightarrow \pi^{+} \pi^{+} \pi^{-}(n)$ was carried out by the CLAS Collaboration at Jefferson Lab. A tagged-photon beam with energies in the 4.8 to $5.4 \mathrm{GeV}$ range, produced through bremsstrahlung from a $5.744 \mathrm{GeV}$ electron beam, was incident on a liquid-hydrogen target. A partial wave analysis was performed on a sample of 83000 events, the highest such statistics to date in this reaction at these energies. The main objective of this study was to look for the photoproduction of an exotic $J^{P C}=1^{-+}$resonant state in the 1 to $2 \mathrm{GeV}$ mass range. Our partial wave analysis shows production of the $a_{2}(1320)$ and the $\pi_{2}(1670)$ mesons, but no evidence for the $a_{1}(1260)$, nor the $\pi_{1}(1600)$ exotic state at the expected levels. An upper limit of $13.5 \mathrm{nb}$ is determined for the exotic $\pi_{1}(1600)$ cross section, less than $2 \%$ of the $a_{2}(1320)$ production.

DOI: 10.1103/PhysRevLett.102.102002

The self-interacting nature of the gluon within quantum chromodynamics (QCD) allows for hybrid resonant states with a $(\bar{q} q g)$ configuration, where the gluonic degree of freedom gives rise to a spectrum of additional states outside the constituent quark model (CQM). The observation of gluonic-hybrid hadrons, with an explicit excitation of this gluonic degree of freedom, will be an important test of the predicting power of $\mathrm{QCD}$ at intermediate energies. A signature of states beyond the CQM would be the existence of mesons with quantum numbers that cannot be attained by $(\bar{q} q)$ mesons (so-called "exotics"). In particular, the lowest lying $(\bar{q} q g)$ state is predicted to have $J^{P C}=1^{-+}$ [1-4], and mass near $1.9 \mathrm{GeV}$. A more recent calculation on the lattice using lighter quark masses predicts a mass at $1.74 \mathrm{GeV}$ [5].

Here we report on a search for exotic mesons decaying to three charged pions. This channel was chosen for its simplicity, since only a few decay channels are available to this final state. In addition, although the dominant decay mode of the lightest $J^{P C}=1^{-+}$state is predicted to be into
PACS numbers: 13.60.Le, 12.38.Qk, 12.39.Mk, 13.25.Jx

an $S$-wave and $P$-wave meson, such as the $b_{1}(1235) \pi$ or $f_{1}(1285) \pi$ [6], the three-pion final state in the $\rho$ decay channel could be non-negligible [7-9].

There is also evidence for an exotic $\pi_{1}(1600)$ state in the reaction $\pi^{-} p \rightarrow \pi^{+} \pi^{+} \pi^{-} p$ at $18 \mathrm{GeV}$ by the Brookhaven E852 experiment [10,11]. Furthermore, the existence of the $\pi_{1}(1600)$ has been confirmed by the same experiment in other channels such as $\eta^{\prime} \pi$ [12]. The VES Collaboration also reported an exotic signal in the $\eta^{\prime} \pi^{-}$channel in the same mass region [13]. However, a more recent analysis of a higher statistics sample from E852 $3 \pi$ data, claims to find no evidence for $\pi_{1}(1600)$ production [14]. All these results were obtained by pion beam experiments.

It has long been anticipated that photoproduction may be a better production mechanism for exotic mesons $[6,8,15-$ 17]. If the $\pi_{1}(1600)$ state couples to $\rho \pi$, then this state should also be produced with a photon beam through $\pi$ exchange via the vector-meson-dominance model [18]. However, the use of photon beams as probes for exotic 
meson production has not been fully explored so far, and the existing data on multiparticle final states are sparse. Photoproduction of mesons in multipion final states has been reported in three previous experiments: a SLAC 40in. hydrogen bubble chamber experiment [19] at $4.3 \mathrm{GeV}$, a CERN hydrogen experiment [20] between 25 and $70 \mathrm{GeV}$, and a SLAC 1-meter hybrid bubble chamber experiment $[21,22]$ around $19 \mathrm{GeV}$. All of these experiments lacked the statistics required for a full partial wave analysis (PWA). For example, Condo et al. [22] showed that the three-pion spectrum in the low-mass region (below $1.5 \mathrm{GeV}$ ) is dominated by $a_{2}(1320)$ production, and found no clear evidence for $a_{1}(1260)$ production. In the high-mass region (1.5 to $2 \mathrm{GeV}$ ), based on an angular distribution analysis, this same group claimed evidence for a narrow state at $1.775 \mathrm{GeV}$ with possible $J^{P C}=1^{-+}, 2^{-+}$, or $3^{++}$assignments. From the analysis of four pion events, Ref. [20] reported two peaks, one at the mass of the $a_{2}(1320)$ and another at around $1.75 \mathrm{GeV}$, in the $\rho \pi$ state recoiling off the proton and the remaining pion.

The present experiment was performed at Jefferson Laboratory during the 2001 running period of CLAS. Details of the design and operation of CLAS and its components may be found in Ref. [23] and references within. The experiment ran with a fixed electron beam energy of $5.744 \mathrm{GeV}$. A tagged-photon beam, with a flux of $5 \times 10^{7}$ photons/s produced via bremsstrahlung from a $3 \times 10^{-4}$ radiation lengths radiator, was incident on a liquid-hydrogen target contained in a cylindrical cell $18 \mathrm{~cm}$ in length. The running conditions were optimized for meson production recoiling off a neutron: the CLAS torus magnet field was reduced to half its maximum current, and the target pulled back $1 \mathrm{~m}$ from the nominal center of CLAS position. Only events with tracks coming from the target material that were in time within $1 \mathrm{~ns}$ with a beam photon with energy between 4.8 and $5.4 \mathrm{GeV}$ were selected.

The three pions in the reaction $\gamma p \rightarrow \pi^{+} \pi^{+} \pi^{-} n$ were identified by the time-of-flight detector, and the neutron was identified through missing mass (Fig. 1). The reconstructed mass of the neutron was $942.2 \mathrm{MeV}$ with a width of $25.1 \mathrm{MeV}$. As shown in Figs. 2(a) and 2(b), the various $n \pi$ invariant mass spectra show clear production of baryon resonances recoiling off a two-pion system, consistent with the process shown in Fig. 3(b). The $n \pi^{-}$invariant mass [Fig. 2(a)] shows a peak at the mass of the $\Delta(1232)$, while the $n \pi^{+}$effective mass distribution [Fig. 2(b)] shows enhancements around the mass of $N^{*}(1520 / 1535)$ and $N^{*}(1650 / 1675 / 1680)$, with a smaller contribution from $\Delta(1232)$ production.

The observed squared four-momentum transfer distribution $-t$ between the incoming photon beam and the produced three-pion system follows an exponential of the form $f(t)=a e^{-b|t|}$. This form is consistent with peripheral production of the three-pion system recoiling off the neu-

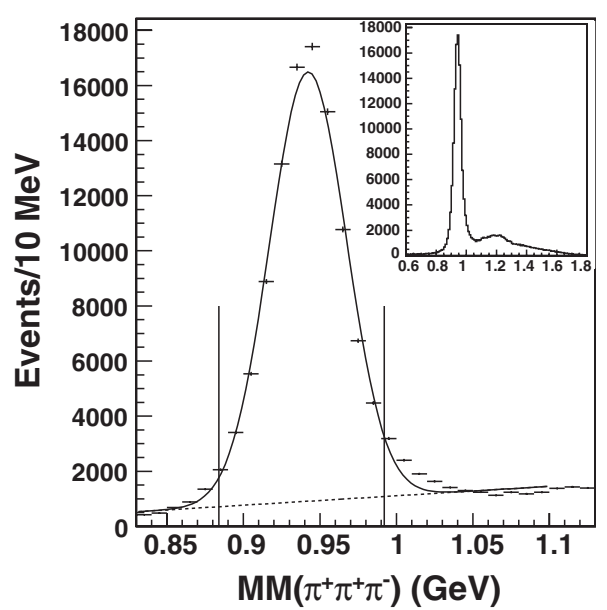

FIG. 1. Missing mass of the three-pion system. The distribution was fit to a Gaussian plus a linear background (solid curve). The inset shows a wider scale. The region between the vertical lines at 0.884 and $0.992 \mathrm{GeV}$ identified the neutron.

tron and is consistent with the production mechanism shown in Fig. 3(a). For the exponential slope constant $b$, we obtained a fitted value of $3.02 \mathrm{GeV}^{-2}$. To enrich the sample of peripheral events, we required $|t| \leq 0.4 \mathrm{GeV}^{2}$. To further enhance the mesonic sample relative to the baryonic background, only events with forward-going $\pi^{+}$'s $\left[\theta_{\mathrm{Lab}}\left(\pi^{+}\right) \leq 30^{\circ}\right]$ were selected. This requirement, as determined from simulation, is necessary to remove most of the baryonic background without significantly affecting the mesonic sample. After all the former selec-
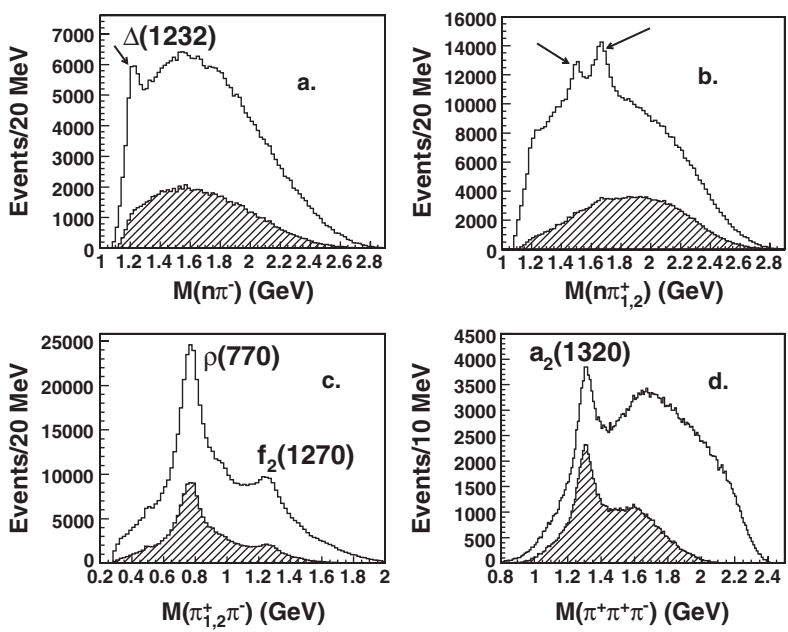

FIG. 2. Mass spectra. (a) $n \pi^{-}$invariant mass, (b) $n \pi^{+}$invariant mass [the left arrow indicates $N^{*}(1520 / 1535)$ resonances and the right arrow $N^{*}(1650 / 1675 / 1680)$ resonances], (c) $\pi^{+} \pi^{-}$invariant mass, and (d) $\pi^{+} \pi^{+} \pi^{-}$invariant mass. The hatched areas are the distributions of remaining events after the momentum transfer and $\pi^{+}$laboratory angle selections. The histograms in (b) and (c) are filled twice for each event, once for each $\pi^{+}$. 

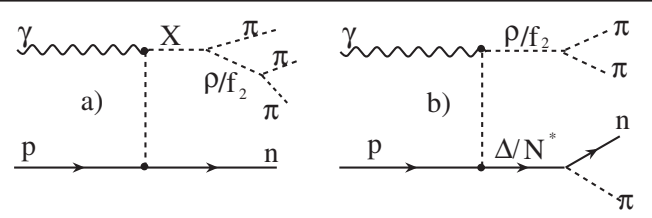

FIG. 3. (a) Peripheral meson production, (b) baryon resonance background processes.

tions were applied, about 83000 events remained as input to the PWA.

The $\pi^{+} \pi^{-}$invariant mass distribution, Fig. 2(c), shows enhancements at the mass of the $\rho(770)$ and $f_{2}(1270)$. The $3 \pi$ invariant mass spectrum, Fig. 2(d), shows a clear peak around the mass of the $a_{2}(1320)$ meson. It also shows a broad enhancement in the $1600-1700 \mathrm{MeV}$ mass region.

A PWA, based on the isobar model, was performed to determine the spin, parity, and charge conjugation $\left(J^{P C}\right)$ of the three-pion system. The three-body decay of the meson was described by a sequence of two-body intermediate decays [Fig. 3(a)]. States with definite $J^{P C}$ of the mesonic system were combined as eigenstates of reflection through the production plane (reflectivity) according to the formalism described in Refs. [24-27]. The expected number of events per mass bin were written as a sum of production and decay amplitudes. To obtain the observed number of events per bin, the acceptance of the detector was determined by normalizing to the Monte Carlo sample. The model used the same $t$ distribution as determined from the data, and the events were simulated in a GEANT-based model of the CLAS detector. A maximum likelihood fit to each mass bin was done with a set of input partial waves. Many different sets of waves were tried during the analysis. The meson spin-density matrix was approximated by a rank 2 matrix due to limited statistics and the large number of parameters required for the fit. The rank of the spindensity matrix represents the number of independent spin components in the initial and final states necessary to describe the interaction. Parity conservation reduces the number of independent components to four.

No baryon resonance partial waves were used and an isotropic noninterfering background wave was included, which is appropriate, since the events were dominated by meson production after the kinematic selection cuts. We separated the PWA in two regions (at $1.36 \mathrm{GeV}$ ) to account for the different goals in the analysis (the observation of a well-known state: the $a_{2}(1320)$ and to determine the components of the $1600 \mathrm{MeV}$ region). We limited the waves in the low-mass region because the higher orbital angular momentum $(L)$ waves do not contribute at low mass. Below $1.36 \mathrm{GeV}$, no $f_{2} \pi$ wave was included as its mass is lower than the nominal threshold. Contributions from $m^{\epsilon}=0^{+}, 0^{-}$waves were found to be small, and these waves are therefore not included in the final wave set. The final list of waves that provided the best stable fit is shown in Table I. The quality of the PWA fit was checked by
TABLE I. Set of partial waves used in the PWA. $J, P, C$ refers to the spin, parity, charge-conjugation of the three-pion system. $m, \epsilon$ are the spin-projection and reflectivity quantum numbers. $L$ is the orbital angular momentum of the decay. Isobar is the intermediate meson that decays to two pions. Note: Background ("Bg") refers to an isotropic, noninterfering wave that is meant to accommodate events that are not described by the interfering waves.

\begin{tabular}{lcccrc}
\hline \hline$\pi^{+} \pi^{+} \pi^{-}$mass & $J^{P C}$ & $m^{\epsilon}$ & $L$ & Isobar & \# Waves \\
\hline $1.0-1.36 \mathrm{GeV}$ & $1^{++}$ & $1^{ \pm}$ & 0,2 & $\rho(770)$ & 4 \\
& $2^{++}$ & $1^{ \pm}, 2^{ \pm}$ & 2 & $\rho(770)$ & 4 \\
13 waves & $1^{-+}$ & $1^{ \pm}$ & 1 & $\rho(770)$ & 2 \\
(48 parameters) & $2^{-+}$ & $1^{ \pm}$ & 1 & $\rho(770)$ & 2 \\
& "Bg" & & & & 1 \\
1.36-2.0 GeV & $1^{++}$ & $1^{ \pm}$ & 0,2 & $\rho(770)$ & 4 \\
& $1^{++}$ & $1^{ \pm}$ & 1 & $f_{2}(1270)$ & 2 \\
31 waves & $2^{++}$ & $1^{ \pm}, 2^{ \pm}$ & 2 & $\rho(770)$ & 4 \\
(120 parameters) & $1^{-+}$ & $1^{ \pm}$ & 1 & $\rho(770)$ & 2 \\
& $2^{-+}$ & $1^{ \pm}, 2^{ \pm}$ & 1 & $\rho(770)$ & 4 \\
& $2^{-+}$ & $1^{ \pm}$ & 3 & $\rho(770)$ & 2 \\
& $2^{-+}$ & $1^{ \pm}, 2^{ \pm}$ & 0 & $f_{2}(1270)$ & 4 \\
& $2^{-+}$ & $1^{ \pm}$ & 2 & $f_{2}(1270)$ & 2 \\
& $3^{++}$ & $1^{ \pm}$ & 2 & $\rho(770)$ & 2 \\
& $3^{++}$ & $1^{ \pm}$ & 1 & $f_{2}(1270)$ & 2 \\
& "Bg” & & & & 1 \\
\hline \hline
\end{tabular}

comparing various mass and angular distributions from the observed data and the predictions of the PWA results, i.e., weighting the Monte Carlo events according to the PWA fit. Qualitatively good agreement was observed between these two sets [28].

Results from the PWA are shown in Figs. 4 and 5. The two most prominent waves are $J^{P C}=2^{++}$and $2^{-+}$ [Figs. 4(c) and 4(d)]. The $a_{2}(1320)$ is observed in the $(\rho \pi)_{D}$ wave at the expected mass and width. For the $a_{2}$ cross section, we obtained a value of $0.81 \pm 0.25 \mu \mathrm{b}$. The uncertainty includes both detector systematics and variation of PWA results estimated by using different wave sets and starting parameters. The result is in rough agreement with the previous measurement of $1.14 \pm 0.57 \mu b$ obtained at $5.25 \mathrm{GeV}$ by Ballam [29] and $1.71 \pm 0.86 \mu b$ obtained at 4.7 to $5.8 \mathrm{GeV}$ by Eisenberg [30].

The $\pi_{2}(1670)$ is observed clearly in the $\left(f_{2} \pi\right)_{S}$ decay mode [Fig. 5(b)]. In the remaining $\pi_{2}$ decay modes: $(\rho \pi)_{P, F}$ [Fig. 5(a)], and $\left(f_{2} \pi\right)_{D}$, the signal looks broad and distorted in the low-mass region. This is most likely due to the incomplete set of waves in the fit, and is indicative of leakage from the $a_{2}$. Similar observations were reported by the E852 Collaboration [11,14]. In addition, due to the two different wave sets used in the two fitting regions (Table I), some instability is observed near the border of $1.36 \mathrm{GeV}$ [Figs. 4(b) and 4(d)]. This should be expected as a result of incomplete wave sets. In order to perform PWA using a larger number of waves and a spin- 


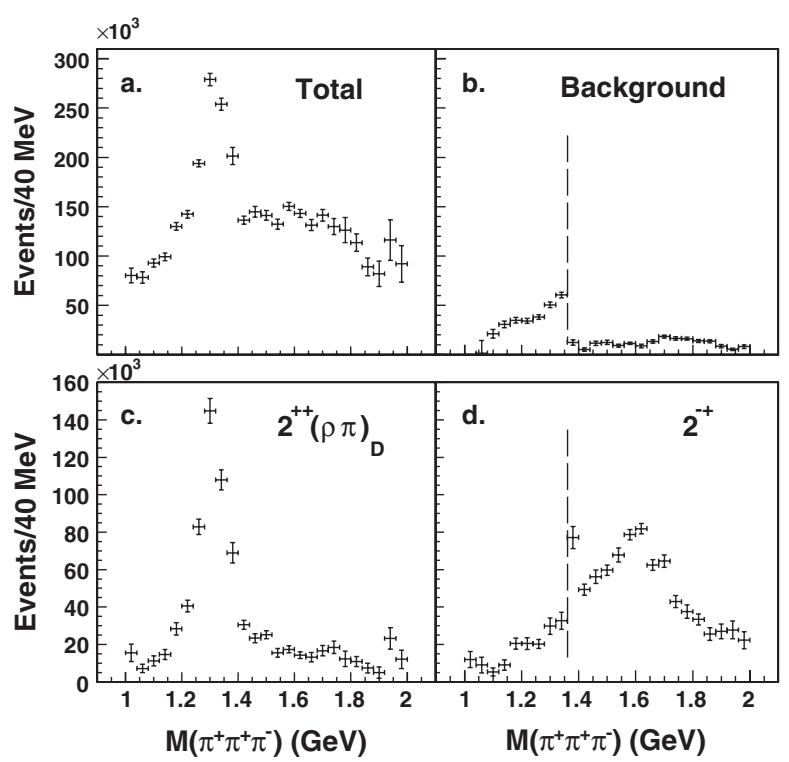

FIG. 4. PWA results: Combined intensities of waves included in the fit. The intensities shown are the sum of the intensities from both ranks of the spin-density matrix: (a) Total intensity, (b) Background intensity, (c) $2^{++}(\rho \pi)_{D}$ intensity, and (d) $2^{-+}$ total intensity. The dashed vertical line in (c) and (d) indicates the border of the two fitting regions. The drop in the isotropic, noninterfering wave ("background") at the boundary, is a consequence of the reduced wave set used at low mass.

density matrix of rank 4, future higher statistics experiments are needed [31].

It is important to note that we see no clear evidence for the $a_{1}(1260)$ in the possible decay mode of the $(\rho \pi)_{S}$ wave [Fig. 5(c)]. This observation agrees with Condo et al. [21]. The event enhancement observed around $1.3 \mathrm{GeV}$ in the noninterfering, flat background waves [Fig. 4(b)] is likely leakage coming from the $a_{2}$ and may demonstrate that the rank of the spin-density matrix is larger than 2 .

We do not observe resonant structure in the exotic $1^{-+}(\rho \pi)_{P}$ partial wave [Fig. 5(d)]. To determine if the $1^{-+}$wave was necessary to better fit our data, we compared PWA fits with and without the $1^{-+}$wave using the likelihood ratio (LR) test $[32,33]$. Using LR statistics, we find that the PWA set of waves including the $1^{-+}$wave fits the data significantly better than a model without the $1^{-+}$ wave. While no clear resonant structure was observed in the $1^{-+}$intensity distribution [Fig. 5(d)], this distribution was used to estimate an upper limit to the $\pi_{1}(1600)$ cross section using the method of Helene [34]. Using the mass of $1597 \mathrm{MeV}$ and the width of $340 \mathrm{MeV}$ as measured by Ref. [12], we estimated an upper limit for the $\pi_{1}(1600)$ of $13.5 \mathrm{nb}$ at a $95 \%$ confidence level, less than $2 \%$ of the $a_{2}(1320)$. Therefore, our results do not agree with the predicted strengths for photoproduction of a $1^{-+}$ gluonic-hybrid meson [6,8,15-17]. Based on Ref. [8], the $\pi_{1}(1600)$ is expected to be produced with a strength near $10 \%$ of the $a_{2}(1320)$. Reference [17] predicts a factor of 5-

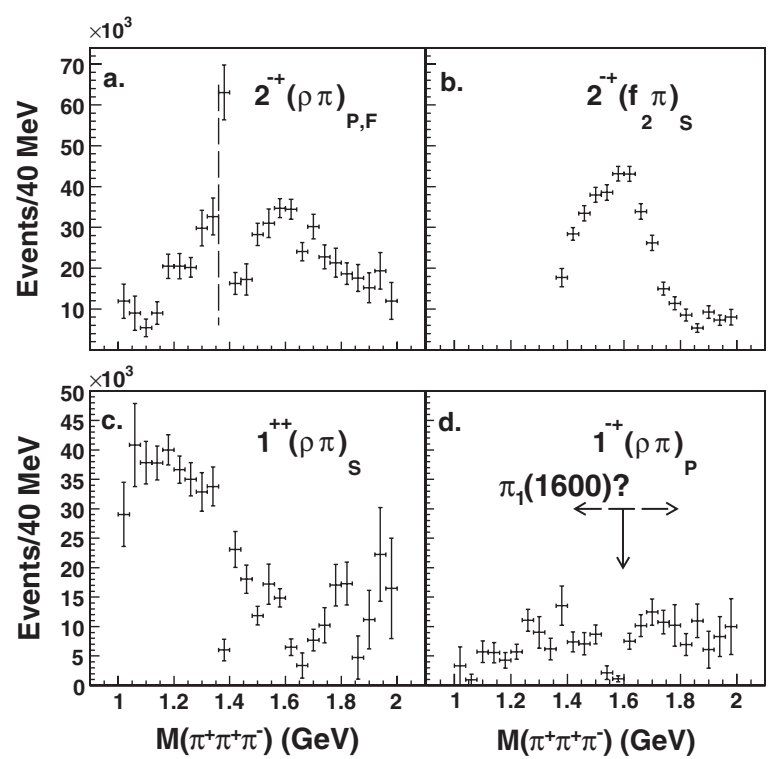

FIG. 5. Partial wave decompositions: (a) $2^{-+}(\rho \pi)_{P, F}$ (the dashed vertical line indicates the border of the two fitting regions), (b) $2^{-+}\left(f_{2} \pi\right)_{S}$, (c) $1^{++}(\rho \pi)_{S}$, and (d) $1^{-+}(\rho \pi)_{P}$ (the vertical and horizontal arrows indicate the mass and width of the $\pi_{1}(1600)$ as reported by Ref. [10]).

10 larger ratio of exotic meson to $a_{2}$ in photoproduction than hadroproduction. This would imply the $\pi_{1}(1600)$ cross section to be on the order of $50 \%$ of $a_{2}$, more than 25 times higher than what we observed. It is possible that the $\pi_{1}(1600)$ reported by Ref. [10] is not a gluonic-hybrid meson, but rather of other nature. Alternatively, the calculated photoproduction cross section of gluonic exotic mesons might be overestimated.

This work was supported in part by the U.S. Department of Energy, the U.S. National Science Foundation, the Italian Istituto Nazionale di Fisica Nucleare, the French Centre National de la Recherche Scientifique, the French Commissariat à l'Energie Atomique, and the Korean Science and Engineering Foundation. Jefferson Science Associates (JSA) operates the Thomas Jefferson National Accelerator Facility for the United States Department of Energy under Contract No. DE-AC05-060R23177.

* Present address: Los Alamos National Laboratory, Los Alamos, NM 87545, USA.

${ }^{\dagger}$ Present address: Ohio University, Athens, OH 45701, USA.

Present address: University of New Hampshire, Durham, NH 03824-3568, USA.

${ }^{\S}$ Present address: Christopher Newport University, Newport News, VA 23606, USA.

"Present address: University of South Carolina, Columbia, SC 29208, USA. 
"Present address: Massachusetts Institute of Technology, Cambridge, MA 02139-4307, USA.

**Present address: Physikalisches Institut der Universitaet Giessen, 35392 Giessen, Germany.

${ }^{\dagger}$ Present address: University of Richmond, Richmond, VA 23173, USA.

\$Present address: Edinburgh University, Edinburgh EH9 3JZ, United Kingdom.

${ }^{\S}$ University of Massachusetts, Amherst, MA 01003, USA.

${ }^{\| \prime}$ Deceased.

[1] C. Bernard et al., Phys. Rev. D 56, 7039 (1997).

[2] K. Juge, J. Kuti, and C. J. Morningstar, Nucl. Phys. B, Proc. Suppl. 63, 326 (1998).

[3] P. Lacock and K. Schilling, Nucl. Phys. B, Proc. Suppl. 73, 261 (1999)

[4] Z. Mei and X. Luo, Int. J. Mod. Phys. A 18, 5713 (2003).

[5] J. N. Hedditch et al., Phys. Rev. D 72, 114507 (2005).

[6] N. Isgur, R. Kokotski, and J. Paton, Phys. Rev. Lett. 54, 869 (1985).

[7] F. E. Close and P. R. Page, Nucl. Phys. B443, 233 (1995).

[8] F. E. Close and P. R. Page, Phys. Rev. D 52, 1706 (1995).

[9] P. R. Page, E. S. Swanson, and A.P. Szczepaniak, Phys. Rev. D 59, 034016 (1999).

[10] G. Adams et al., Phys. Rev. Lett. 81, 5760 (1998).

[11] S. U. Chung et al., Phys. Rev. D 65, 072001 (2002).

[12] E. Ivannov et al., Phys. Rev. Lett. 86, 3977 (2001).

[13] Y. Khokhlov et al., Nucl. Phys. A663, 596 (2000).

[14] A. Dzierba et al., Phys. Rev. D 73, 072001 (2006).
[15] A. Afanasev and P. Page, Phys. Rev. D 57, 6771 (1998).

[16] A. Afanasev and A. Szczepaniak, Phys. Rev. D 61, 114008 (2000).

[17] A. Szczepaniak and M. Swat, Phys. Lett. B 516, 72 (2001).

[18] M. Gell-Mann, Phys. Rev. 125, 1067 (1962).

[19] Y. Eisenberg et al., Phys. Rev. Lett. 23, 1322 (1969).

[20] D. Aston et al., Nucl. Phys. B189, 15 (1981).

[21] G. T. Condo et al., Phys. Rev. D 48, 3045 (1993).

[22] G. T. Condo et al., Phys. Rev. D 43, 2787 (1991).

[23] B. A. Mecking et al., Nucl. Instrum. Methods Phys. Res., Sect. A 503, 513 (2003).

[24] S. U. Chung, Spin Formalism, CERN Yellow Report No. CERN 71-8, 1971; Formulas for Partial Wave Analysis, Version II, BNL report No. QGS-93-05, 1993.

[25] S. U. Chung and T.L. Trueman, Phys. Rev. D 11, 633 (1975).

[26] J.P. Cummings and D.P. Weygand, arXiv:physics/ 0309052.

[27] P. Eugenio, Int. J. Mod. Phys. A 18, 487 (2003).

[28] M. Nozar et al., arXiv:hep-ex/0312037.

[29] J. Ballam et al., Phys. Lett. 30B, 421 (1969).

[30] Y. Eisenberg et al., Phys. Rev. D 5, 15 (1972).

[31] P. Eugenio et al., CLAS Proposal, Jefferson Laboratory Report No. E-04-005, 2004.

[32] Eadie et al., Statistical Methods in Experimental Physics (North-Holland Pub. Co., Amsterdam, 1982), 2nd ed.

[33] F. James and M. Ross, CERN Tech. Report No. 506, 1992.

[34] O. Helene, Nucl. Instrum. Methods 212, 319 (1983). 